\title{
Revisiting the relationship between the submandibular duct, lingual nerve and hypoglossal nerve
}

\author{
C. Günenç Beşer ${ }^{1}$, B. Erçakmak ${ }^{1}$, H.B. Ilgaz ${ }^{1}$, A. Vatansever ${ }^{2}$, M.F. Sargon ${ }^{1}$ \\ ${ }^{1}$ Department of Anatomy, Faculty of Medicine, Hacettepe University, Ankara, Turkey \\ ${ }^{2}$ Department of Anatomy, Faculty of Medicine, Balıkesir University, Balıkesir, Turkey
}

[Received: 17 November 2017; Accepted: 8 January 2018]

Background: The aim of the study was to evaluate the relations between submandibular duct, lingual nerve and hypoglossal nerve for making a reassessment of this area in fresh frozen specimens. Also, the distance between the angle of the mandible and the vertical line drawn from the point where submandibular duct crossed lingual nerve to the base of the mandible was measured to determine a new landmark for neck surgeons.

Materials and methods: Fourteen fresh frozen head and neck specimens were dissected and evaluated. A marginal mandibular incision was made from the mastoid process to the chin.

Results: In 8 cases, lingual nerve was crossing the submandibular duct superiorly; in 5 cases, lingual nerve was crossing the duct infero-medially and in 1 case it was parallel to the duct. In 1 case, lingual nerve subdivided into anterior and posterior branches. In 2 cases, 2 parallel submandibular ducts were found and the lingual nerve was crossing the upper duct from superior. In 1 case, lingual nerve was crossing the duct infero-medially and then it was subdividing into branches superior to mylohyoid. In 12 cases, the course of hypoglossal nerve was classical. In 1 case, hypoglossal nerve crossed the submandibular duct medially and coursed parallel to the tendon of posterior belly of digastric. And in another case, hypoglossal nerve crossed the inferior branch of submandibular duct medially. The other structures in this area were as usual.

Conclusions: The main factor for reducing nerve damage during surgery is the understanding of the anatomy of this area. (Folia Morphol 2018; 77, 3: 521-526)

Key words: lingual nerve, submandibular duct, hypoglossal nerve, anatomy, dissection

\section{INTRODUCTION}

Submandibular duct (SD) is also referred as Wharton's duct and it is the main excretory duct of the submandibular gland. SD begins by numerous branches that leave the submandibular gland anteriorly from the sublingual aspect, coursing deep to the lingual nerve (LN) and medial to the sublingual gland. It bends sharply at the posterior margin of mylohyoid to form the genu of SD, and then it runs forward lateral to hyoglossus and genioglossus and medial to the insertion of mylohyoid to the mandible. Afterwards, it passes between hyoglossus and mylohyoid

Address for correspondence: C. Güneng Beşer, MD, PhD, Department of Anatomy, Faculty of Medicine, Hacettepe University Sihhiye, Ankara, Turkey, tel: +90-312-3052355, fax: +90-312-3107169, e-mail: crngnc5er@gmail.com 
muscles on the genioglossus muscle. It is $4-5 \mathrm{~cm}$ in length, running superior to the hypoglossal nerve (HN) and inferior to the LN. At the anterior border of hyoglossus muscle it is crossed laterally by the LN. This duct drains into a papilla in the floor of the mouth behind the lower incisor tooth, lateral to the lingual frenulum $[16,19]$.

The $L N$ is a branch of the posterior division of mandibular nerve that carries the presynaptic fibres to the submandibular ganglion. The postsynaptic nerve innervates both the submandibular and sublingual glands. It also takes the general sensation of the mucosa of the anterior two-thirds of the tongue, the sublingual mucosa, the mandibular lingual gingiva and the floor of the mouth. LN courses laterally between the medial pterygoid muscle and ramus of mandible, enters the oral cavity at the lower third molar, then it travels across the hyoglossus along the floor of the mouth. It lies on the deep part of the submandibular gland and passes below the SD which crosses it from medial to lateral, then curves upward, forward and medially to enter the tongue. LN is the first key for the glandular excision by the endoscopic intraoral approach $[1,8,16,19]$.

The HN travels just deep to the posterior belly of the digastric muscle and common tendon until it reaches the submandibular triangle. Its location is anterior, deep and medial relative to the submandibular gland. Generally, the nerve has a close relationship to the anterior belly of the digastric muscle. It then ascends anterior to the LN and its genu deep to the mylohyoid muscle. It lies below the deep part of the submandibular gland, SD and LN, with which it communicates. Extreme care should be taken to preserve this important nerve during head and neck surgery $[16,19]$.

The aim of our study was to evaluate the relations between SD, LN and $\mathrm{HN}$ for making a reassessment of this area in fresh frozen specimens. Also, the distance between the angle of the mandible and the vertical line drawn from the point where SN crossed LN to the base of the mandible was measured to determine a new landmark for neck surgeons.

\section{MATERIALS AND METHODS}

In the study, 7 fresh frozen head and neck specimens were used and the dissections were made bilaterally. Totally, 14 specimens' neck regions were dissected. First of all, a marginal mandibular incision was made from the mastoid process to the chin. The

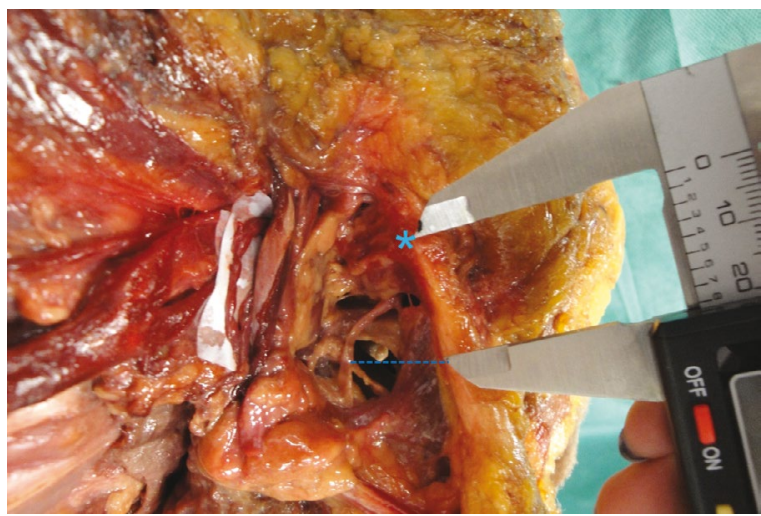

Figure 1. The distance between the angle of mandible and the vertical line drawn from the point where submandibular duct crossed lingual nerve to the base of the mandible; asterisk - the angle of the mandible; dashed line - the vertical line drawn from the point where submandibular duct crossed lingual nerve to the base of the mandible.

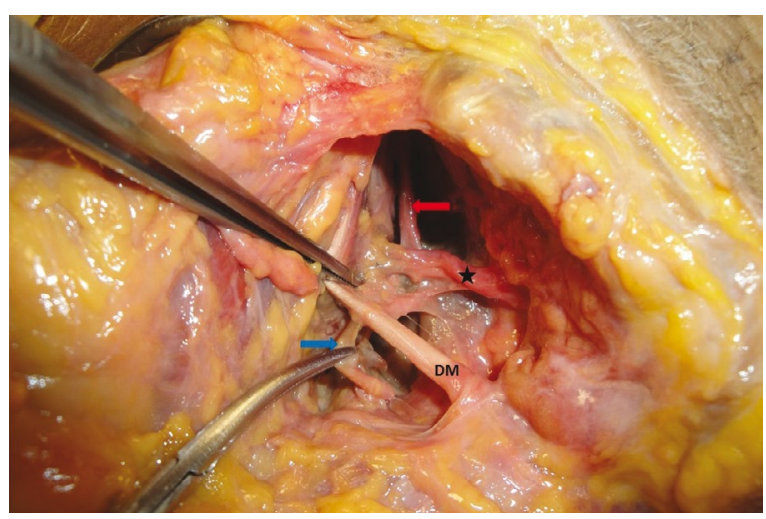

Figure 2. Lingual nerve crossing the submandibular duct superiorly; red arrow — lingual nerve; blue arrow — hypoglossal nerve; asterisk — submandibular duct; DM — digastric muscle, posterior belly.

dissection was standardised in each case. The relationships between the SD, LN and HN were investigated. The distance between the angle of mandible and the vertical line drawn from the point where SD crossed LN to the base of the mandible was also measured with a digital goniometer (150 $\mathrm{mm}$ digital calliper) (Fig. 1). Before each measurement, the correction of the goniometer was done. Statistical analyses were performed by using SPSS 15 .

\section{RESULTS}

In our study, head and neck regions of 7 fresh frozen specimens were dissected bilaterally. Fourteen specimens' SDs, hypoglossal and LNs and their relations were examined. LN was crossing the SD superiorly in 8 cases (Fig. 2); whereas, it was cross- 


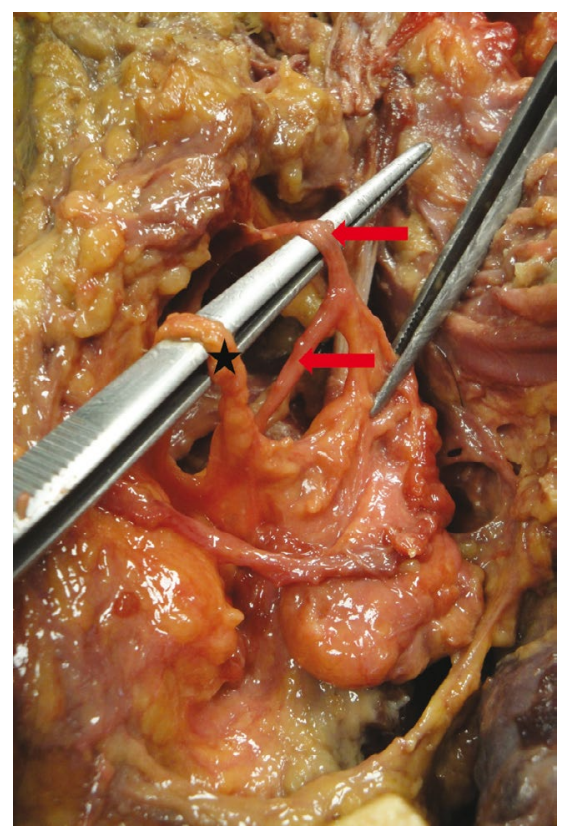

Figure 3. Lingual nerve subdivided into two branches: anterior and posterior. Anterior branch crossed the submandibular duct superiorly within the gland; posterior branch coursed parallel to the duct; red arrows - lingual nerve, anterior and posterior branches; asterisk — submandibular duct.

ing the duct infero-medially in 5 cases. In 1 case, LN was parallel to the gland and coursed from the supero-medial aspect of it. LN was subdivided into 2 branches while entering the gland in 1 case; anterior and posterior branches. Anterior branch crossed the SD superiorly within the gland; posterior branch coursed parallel to the duct (Fig. 3). In 2 cases (from different fresh frozen specimens), 2 parallel SDs were found and the LN was crossing the upper duct from superior (Fig. 4). Right side of 1 specimen had 2 parallel SDs and left side of it had 1 large duct which was parallel to the LN coursing supero-medially. In 1 case, LN was crossing the SD infero-medially and then it was subdividing into 2 branches superior to mylohyoid (Fig. 5).

The findings of the study were summarised in Table 1.

The distance between the angle of the mandible and the vertical line drawn from the point where SD crossed LN to the base of the mandible was measured in 13 fresh frozen specimens. In 1 specimen, LN was parallel to the SD, so there was not a cross between them. The mean distance was found $30.231 \pm 8.78$ $\mathrm{mm}$ (range 17-45 $\mathrm{mm}$ ).

In 12 cases, the course of HN was classical; it was passing deep to the posterior belly of the digastric

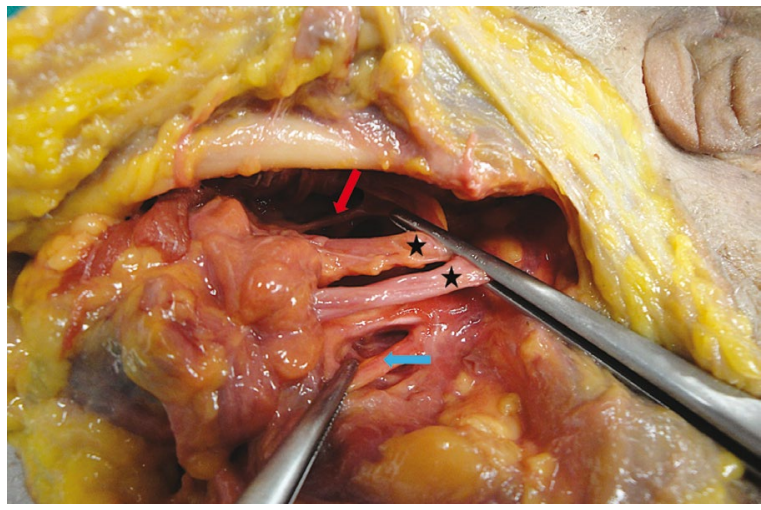

Figure 4. Two parallel submandibular ducts, lingual nerve crossing the upper duct from superior; red arrow - lingual nerve; asterisks — submandibular duct; blue arrow — hypoglossal nerve.

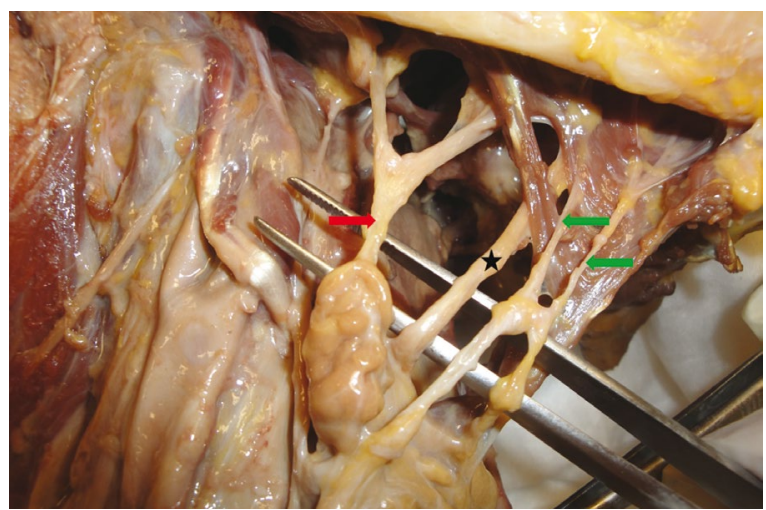

Figure 5. Lingual nerve crossing the submandibular duct inferomedially and then dividing into two branches; red arrow - lingual nerve; asterisk — submandibular duct; green arrows - mylohyoid nerves.

muscle. It was placing anterior, deep and medial to the submandibular gland. It had a close relationship to the anterior belly of the digastric muscle. Then it ascended anterior to the LN. In 1 case, HN crossed the SD medially and coursed parallel to the tendon of posterior belly of digastric. And in another case, HN crossed the inferior branch of SD medially (Fig. 6).

The other structures in this area were as usual.

\section{DISCUSSION}

The most frequent disorders that require surgical excision of the submandibular gland are chronic inflammation of the gland with or without sialolithiasis, followed by benign tumours (pleomorphic adenoma, neurinoma, haemangioma, lymphangioma and lipoma) and malignant tumours (adenoid cystic carci- 
Table 1. The findings of the dissections in each side of the specimens

\begin{tabular}{|c|c|c|c|c|}
\hline Specimens & & Relations between LN and SD & Cross & HN \\
\hline \multirow[t]{2}{*}{1} & $\mathrm{R}$ & LN superiorly crossed SD & $21 \mathrm{~mm}$ & $\mathrm{~N}$ \\
\hline & $\mathrm{L}$ & LN superiorly crossed SD & $17 \mathrm{~mm}$ & $\mathrm{~N}$ \\
\hline \multirow[t]{2}{*}{2} & $\mathrm{R}$ & LN superiorly crossed SD & $24 \mathrm{~mm}$ & $\mathrm{~N}$ \\
\hline & L & $\begin{array}{l}\text { LN subdivided into two; anterior and posterior. } \\
\text { Anterior branch superiorly crossed SD within the gland, } \\
\text { posterior branch was parallel to SD }\end{array}$ & $27 \mathrm{~mm}$ & N \\
\hline \multirow[t]{2}{*}{3} & $\mathrm{R}$ & LN infero-medially crossed SD & $34.2 \mathrm{~mm}$ & HN crossed SD medially \\
\hline & $\mathrm{L}$ & $\begin{array}{l}\text { Two parallel SDs, LN superiorly crossed } \\
\text { the upper SD }\end{array}$ & $34 \mathrm{~mm}$ & $\begin{array}{l}\text { HN crossed the inferior branch } \\
\text { of } S D \text { medially }\end{array}$ \\
\hline \multirow[t]{2}{*}{4} & $\mathrm{R}$ & $\begin{array}{l}\text { Two parallel SDs; inferior duct was narrower, } \\
\text { LN superiorly crossed the upper duct }\end{array}$ & $28 \mathrm{~mm}$ & $\mathrm{~N}$ \\
\hline & $\mathrm{L}$ & One large SD. LN was parallel to it and coursed supero-medially & No cross & $\mathrm{N}$ \\
\hline \multirow[t]{2}{*}{5} & $\mathrm{R}$ & $\begin{array}{l}\text { LN infero-medially crossed SD. } \\
\text { LN subdivided into branches }\end{array}$ & $45 \mathrm{~mm}$ & $\mathrm{~N}$ \\
\hline & $\mathrm{L}$ & LN infero-medially crossed SD & $44 \mathrm{~mm}$ & $\mathrm{~N}$ \\
\hline \multirow[t]{2}{*}{6} & $\mathrm{R}$ & LN infero-medially crossed SD & $37 \mathrm{~mm}$ & $\mathrm{~N}$ \\
\hline & L & LN infero-medially crossed SD & $36 \mathrm{~mm}$ & $\mathrm{~N}$ \\
\hline \multirow[t]{2}{*}{7} & $\mathrm{R}$ & LN superiorly crossed SD & $25 \mathrm{~mm}$ & $\mathrm{~N}$ \\
\hline & L & LN superiorly crossed SD & $22 \mathrm{~mm}$ & $\mathrm{~N}$ \\
\hline
\end{tabular}

HN — hypoglossal nerve; L — left side; LN — lingual nerve; $\mathrm{N}$ — Normal; $\mathrm{R}$ — right side; SD — submandibular duct

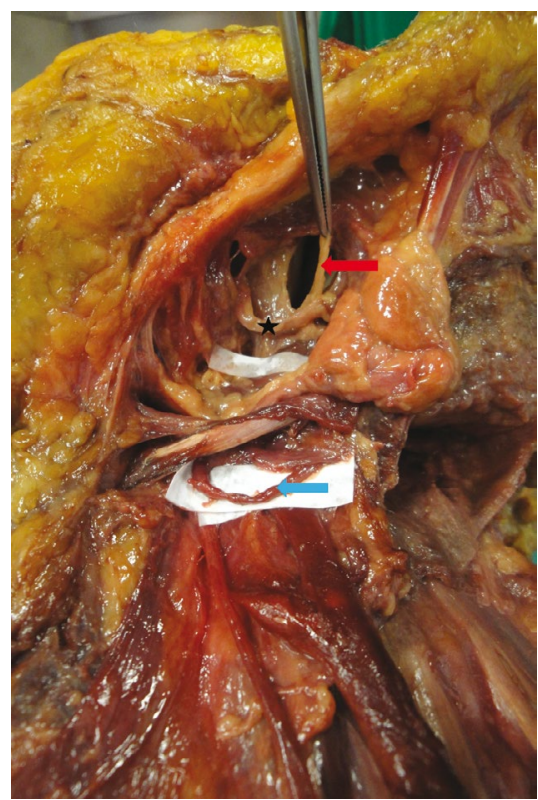

Figure 6. The classical course of the hypoglossal nerve; red arrow — lingual nerve; asterisk — submandibular duct; blue arrow hypoglossal nerve.

noma, adenocarcinoma, mucoepidermoid carcinoma, metastatic squamous cell carcinoma). Excision of the submandibular gland is regarded as a minor surgical operation. Nevertheless, careful anatomic dissection and protection of the nerves in this area are required. These nerves are the marginal mandibular branch of the facial nerve, LN and HN. LNs and HNs are large nerves with coherently standard anatomic courses. But the variations of these two nerves should be well known by the surgeon to minimise the complications of this surgical procedure. $\mathrm{LN}$ and $\mathrm{HN}$ can be damaged if there is a severe inflammatory reaction or massive bleeding. There is no consensus about the routine identification of the nerves and the skin incision [13]. Because of the long and twisted course of the LN, it can be damaged by a variety of procedures such as; tonsillectomies, third molar extractions, local anaesthesia injections, laryngoscopies, SD explorations and salivary gland surgeries [10].

There are many reports about the frequency of the nerve complications in this type of surgery that varies widely. The experience of the surgeon and the type of the surgical manoeuvre are important factors for surgical complications. The experienced head and neck surgeons showed the lowest rate of complication because they are familiar with the anatomy of this region [13].

In the literature, the entity of double ducts of the submandibular gland was reported $[5,7,20,21]$. The presence of double ducts is important for treating bilateral dry eyes with one transferred submandibular gland through transfer of double ducts [11]. In our study, two parallel SDs were found in 2 cases and the LN was crossing the upper duct from superior. One of the specimens had 2 parallel SDs on the right and had one large duct on the left that was parallel to $L N$ coursing supero-medially. 
Most of the studies describe a loop configuration of the $L N$ around the SD $[1,9,17]$. They claim that the $L N$ passes lateral to the SD, then continues superiorly and anteriorly on its medial side to the surface of hyoglossus [6, 12]. This is known as the classical pattern of the nerve. Mendes et al. [15] found that this situation occurs in $62.5 \%$ of the cadavers; in the remaining $37.5 \% \mathrm{LN}$ crosses above the duct. Hölzle et al. [12] reported that SD runs deep in the floor of the oral cavity without a relationship with the $L N$ in $11.8 \%$ of the cases. Al-Amery et al. [1] found that LN was passing below the SD before rising again toward the ventral surface of the tongue in 12 out of the 13 of the cases; in 1 case the relationship was similar to the findings of Hölzle et al. [12, 15].

In our study, LN was passing below the SD in 5 cases which is relevant to the classical pattern that matches with the findings of Mendes et al. [15] and Al-Amery et al. [1]. And LN was crossing above the SD in 8 cases. There was no significant difference between these conditions. In 1 case, LN subdivided into 2 branches (anterior, posterior) while entering the gland. Anterior branch was crossing above the SD; posterior branch was passing parallel to the duct. In 1 case, LN was crossing below the SD and then it was subdividing into 2 branches superior to mylohyoid.

Mendes et al. [15] reported that there was not a constant position for the intersection point of the SD and LN, but the cross is around the premolar region. Castelli et al. [4] reported that $55 \%$ of the intersections occurred at the level of the third molar tooth or behind it. Klepacek and Skulec [14] identified a constant position of this cross in the close vicinity of the upper surface of the posterior portion of the mylohyoid muscle closely to the inner surface of the mandible within the range of the third permanent molar. In the lateral view, the LN and SD cross courses on the external mandibular surface distally from the retrotrigonal triangle into the region where the mandibular body and mandibular branch are fused [14]. Al-Amery et al. [1] found the mean distance of overlap between these two structures was $6.92 \mathrm{~mm}(1.5-13 \mathrm{~mm})$, most of the loops terminated at between the first and second molar teeth. They also found the mean distance between the $\mathrm{LN}$ and alveolar ridge was $12.36 \mathrm{~mm}$ and the mean distance between the $\mathrm{LN}$ and the lower border of mandible was $12.03 \mathrm{~mm}$ [1].

Tumours of the submandibular gland are usually within the gland, and resection is confined to the gland, surrounding fat and lymph nodes. A curvilinear incision, extending from the midline of the mandible to the mastoid process following the course of the digastric muscles, provides good exposure [3]. Hence, we used the angle of the mandible as a landmark, as we think that this is a practical approach with possible clinical application. In the cervical submandibular gland resection, it is recommended that the incision should be placed at least $3 \mathrm{~cm}$ below the angle of the mandible to constantly avoid injury to the branches of the facial nerve [18]. In our study, the mean distance between the angle of the mandible and the point where SD crosses $L N$ was found $32.91 \pm 9.46 \mathrm{~mm}$ (range 17-45 mm). The variable range of the findings necessitates increasing the number of the cadavers for obtaining a significant result.

Submandibular gland is related to the glossopharyngeal nerve, LN and HN medially. Deep part of the gland is related to the LN superiorly and $\mathrm{HN}$ inferiorly. The first part of the SD lies beneath the LN and above the HN [2]. The LN is located superiorly under the mandible and is attached to the gland by the submandibular ganglion. SD lies deep to the $\mathrm{LN}$ and it should be ligated carefully to avoid nerve injury during surgery. The HN is located inferiorly below the digastric muscle and a venous plexus accompanies it [3].

In our study, the course of HN was classical in 12 cases; it was passing deep to the posterior belly of the digastric muscle and placing anterior, deep and medial to the submandibular gland. It was close to the anterior belly of the digastric muscle. Then it ascended anterior to the LN. In 1 case, HN crossed the SD medially and coursed parallel to the tendon of posterior belly of digastric. And in another case, $\mathrm{HN}$ crossed the inferior branch of SD medially.

Ichimura et al. [13] reported that in 41 resections $\mathrm{LN}$ could not be identified during the surgery. Identification of the $\mathrm{LN}$ will reduce the incidence of temporary paraesthesia. When the posterior border of the mylohyoid muscle is retracted anteriorly SD, $\mathrm{LN}$ and $\mathrm{HN}$ are easily identified. There is controversy about the benefit of routine nerve identification at surgery. Some of the surgeons are consistent about the identification of the nerve is the only certain method to avoid injury; however, other surgeons claim that it contains more risk to identify the nerve than to use a method of excision that will safeguard the nerve, because classical visualisation may cause neurapraxia and altered conductivity that leads to paresis [13]. 
This study may be useful for the sialography, sialoendoscopy and submandibular gland transfer. The technical facility and good knowledge of anatomy of the surgeon is important to reduce the injury to the nerves in this area. There is a wide variation in the course of $L N$ up to its intersection with the SD. If the surgical procedures were performed without enough appreciation of the relationship between $\mathrm{LN}$ and SD, the damage potentiality to the LN will be increased as it is intertwined with the SD [1]. This study was limited by the number of the cadavers available so the findings may not possibly present the general population.

\section{CONCLUSIONS}

Although the anatomical courses of SD, LNs and HNs are sufficiently known, there are few conclusive reports on their anatomical variants. We defined cases where the LN was crossing SD superiorly (8 cases), infero-medially ( 5 cases) or LN was parallel to SD (1 case). Also, we found one LN subdivided into 2 branches which had different courses (1 case), and we defined 2 cases with 2 parallel SDs. And the course of the HN was unusual in 2 cases. Even right and left sides of a specimen had different variations. Our study showed that the anatomical variations of these structures are frequent and diversified. The routine identification of the $\mathrm{LN}$ and $\mathrm{HN}$ is used to avoid injury by many surgeons. Appreciation of these variations is also very important in assessing new diagnostic and therapeutic techniques [11]. The limitation of our study is the number of the cadavers available to precisely define the course of these structures.

The surgeons use many different surgical techniques to reach the submandibular region. There is no consensus about the routine identification of the nerves and the skin incision. In this study, the distance between the angle of the mandible and the point where SD crossed LN was measured to determine a new landmark for neck surgeons. The measurements were very variable, so increasing the number of the cadavers would provide a more precise landmark for the surgeons.

This study was presented in the National Anatomy Congress 2016, llgaz, Turkey as a poster presentation.

All procedures performed in this study involving human cadavers were in accordance with the ethical standards of the institutional and national research committee and with the 1964 Helsinki declaration and its later amendments or comparable ethical standards.

\section{REFERENCES}

1. Al-Amery SM, Nambiar $\mathrm{P}, \mathrm{Naidu} \mathrm{M}$, et al. Variation in lingual nerve course: a human cadaveric study. PLoS One. 2016; 11(9): e0162773, doi: 10.1371/journal. pone.0162773, indexed in Pubmed: 27662622.

2. Bademci G, Yaşargil MG. Microsurgical anatomy of the hypoglossal nerve. J Clin Neurosci. 2006; 13(8): 841-847, doi: 10.1016/j.jocn.2005.12.028, indexed in Pubmed: 16935514.

3. Carlson GW. The salivary glands. Embryology, anatomy, and surgical applications. Surg Clin North Am. 2000; 80(1): 261-73, xii, indexed in Pubmed: 10685152.

4. Castelli WA, Huelke DF, Celis A. Some basic anatomic features in paralingual space surgery. Oral Surg Oral Med Oral Pathol. 1969; 27(5): 613-621, indexed in Pubmed: 5253540.

5. Chen SR, Li WB, Zhu W. The double ducts of the submandibular gland - 1 Case. Chin J Clin Anat. 2000; 18: 88.

6. Erdogmus S, Govsa F Celik S. Anatomic position of the lingual nerve in the mandibular third molar region as potential risk factors for nerve palsy. J Craniofac Surg. 2008; 19(1): 264-270, doi: 10.1097/scs.0b013e31815c9411, indexed in Pubmed: 18216699.

7. Gadodia A, Seith A, Neyaz Z, et al. Magnetic resonance identification of an accessory submandibular duct and gland: an unusual variant. J Laryngol Otol. 2007; 121(9): e18, doi: 10.1017/S0022215107008602, indexed in Pubmed: 17517164

8. Guerrissi JO, Taborda G. Endoscopic excision of the submandibular gland by an intraoral approach. J Craniofac Surg. 2001; 12(3): 299-303, indexed in Pubmed: 11358106 .

9. Halim A. Human anatomy: regional and clinical for dental students: I.K. International publishing house pvt. Limited. 2008.

10. Hong SS, Yheulon CG, Sniezek JC. Salivary gland surgery and medical malpractice. Otolaryngol Head Neck Surg. 2013; 148(4): 589-594, doi: 10.1177/0194599813475566, indexed in Pubmed: 23380759.

11. Horsburgh A, Massoud TF. The salivary ducts of Wharton and Stenson: analysis of normal variant sialographic morphometry and a historical review. Ann Anat. 2013; 195(3): 238-242, doi: 10.1016/j.aanat.2012.11.003, indexed in Pubmed: 23279725.

12. Hölzle FW, Wolff KD. Anatomic position of the lingual nerve in the mandibular third molar region with special consideration of an atrophied mandibular crest: an anatomical study. Int J Oral Maxillofac Surg. 2001; 30(4): 333-338, doi: 10.1054/ijom.2001.0064, indexed in Pubmed: 11518358.

13. Ichimura K, Nibu K, Tanaka T. Nerve paralysis after surgery in the submandibular triangle: review of University of Tokyo Hospital experience. Head Neck. 1997; 19(1): 48-53, indexed in Pubmed: 9030945.

14. Klepácek I, Skulec R. Relation between lingual nerve, submandibular gland duct and mandibular body in the sublingual space. Acta Chir Plast. 1993; 36(1): 26-27, indexed in Pubmed: 7618397.

15. Mendes MB, de Carvalho Leite Leal Nunes CM, de Almeida Lopes MC. Anatomical relationship of lingual nerve to the region of mandibular third molar. J Oral Maxillofac Res. 2013; 4(4): e2, doi: 10.5037/jomr.2013.4402, indexed in Pubmed: 24478912 .

16. Holsinger F, Bui D. Anatomy, function, and evaluation of the salivary glands. Salivary Gland Disorders. 2007: 1-16, doi: 10.1007/978-3-540-47072-4_1.

17. Parker S. MRCS Applied Basic Science and Clinical Topics: JP Medical Limited. 2013.

18. Smith AD, Elahi MM, Kawamoto HK, et al. Excision of the submandibular gland by an intraoral approach. Plast Reconstr Surg. 2000; 105(6): 2092-2095, indexed in Pubmed: 10839409.

19. Standring S. Gray's anatomy: the anatomical basis of clinical practice, 40th edition. Churchill Livingstone-Elsevier, London 2008: 505-507.

20. Towers JF. Duplication of the submandibular salivary duct. Oral Surg Oral Med Oral Pathol. 1977; 44(2): 326, indexed in Pubmed: 268586.

21. Zhang L, Xu H, Cai Zg, et al. Clinical and anatomic study on the ducts of the submandibular and sublingual glands. J Oral Maxillofac Surg. 2010; 68(3): 606-610, doi: 10.1016/j.joms.2009.03.068, indexed in Pubmed: 20171479 . 